\title{
Corticosteroid-induced psychiatric disorders: genetic studies are needed
}

\author{
Florence Thibaut ${ }^{1}$ (1)
}

Published online: 6 August 2019

○) Springer-Verlag GmbH Germany, part of Springer Nature 2019

Glucocorticoids are steroid hormones, secreted by the adrenal glands, that bind with high affinity to brain mineralocorticoid receptors (MR) and lower affinity to glucocorticoid receptors (GR). GR are widely distributed throughout the brain, whereas MR are mainly found in the hippocampus. Cortisol exerts predominantly its tonic influences via hippocampal MR, whereas feedback actions at the level of the pituitary as well as actions at the amygdala level are mediated by GR. In addition, glucocorticoid responsive elements are found in the regulatory regions of many genes in the brain. Glucocorticoids are involved in different processes such as glucose metabolism, inflammation, immunity, etc. The stress responsive hypothalamic pituitary adrenal (HPA) axis regulates glucocorticoid production and is implicated in the pathophysiology of anxiety and depression as well as in cognitive functioning or the development and relapse of psychotic disorders. Major depression may be associated with a dysregulation of MR and/or GR within the HPA axis; elevated HPA activity could be more often associated with depression with psychotic features. Elevated cortisol secretion has also been positively linked with symptom severity in people with psychotic disorders. Antiglucocorticoid and related drugs that target the HPA axis may be useful for the treatment of people with psychosis and were also studied in the treatment of depressive disorders or in the improvement of cognitive dysfunction associated with these disorders [1]. Moreover, inflammation plays a significant role in the pathophysiology of major psychiatric disorders.

Despite their well-known potent anti-inflammatory and immuno-suppressant properties in many medical conditions, corticosteroid treatments are often associated with neuropsychiatric complications, which remain poorly understood.

Florence Thibaut

florence.thibaut@aphp.fr

1 University Hospital Cochin, Paris University, INSERM U1266, Institute of Psychiatry and Neurosciences, Paris, France
Corticosteroid treatments are mainly glucocorticoids: the most frequently used are hydrocortisone, cortisone, prednisone and its metabolite prednisolone, methylprednisolone or, dexamethasone (which has a longer half-life and higher anti-inflammatory properties). Up to $10 \%$ of medical/surgical inpatients received corticosteroids and $1-3 \%$ of the general adult population was maintained on long-term corticosteroids (at least 3 months) according to a British prevalence study. In the fifties, several authors reported that even after small doses of cortisone, almost all patients experienced some psychic change: increased joviality, optimism; psychostimulant effects were often observed [2]. Affective disorders (depression or mania), irritability, anxiety are the most common disorders observed; psychotic symptoms are frequently associated with mood disorders. Delirium as well as cognitive deficits (especially deficits in verbal or declarative memory) are also observed during both short-term and long-term treatment. In a metaanalysis of uncontrolled studies, Lewis and Smith [3] reported a weighted average of $6 \%$ of severe depression, mania and psychosis (including hallucinations) and a mean of $28 \%$ of mild to moderate psychiatric symptoms. As often observed in clinical cases of hypercortisolism (Cushing syndrome), major depression is often observed with long-term therapy, whereas manic symptoms are more often observed with pulse-dose or shortterm therapy. Yet, differentiating between corticosteroidinduced psychiatric disorders and central nervous system manifestations of inflammatory or immune diseases is not easy. Mood elevation often associated with corticosteroids can contribute to abuse of corticosteroids. Case reports of catatonia, panic attacks, or reversible dementia were observed. Corticoids may also precipitate suicidal behavior in depressive patients. Finally, corticosteroid use, especially the inhaled agents, is higher in obese than in non-obese individuals. Lewis and Smith [3] reported a median time to onset of neuropsychiatric adverse effects of 11.5 days. Most adverse effects not only are observed within days after treatment initiation but also may occur after treatment cessation 
[2]. Dosage appears as the most significant risk factor. In fact, the risk of psychiatric reaction is tenfold higher when $80 \mathrm{mg} /$ day of prednisone is compared to $40 \mathrm{mg} /$ day (Boston collaborative study, see [2]). High doses of hydrocortisone (200-400 mg/day) prescribed in acute adrenal crisis may also be associated with mania. Female gender is also more often associated with depression. Although small studies did not support past psychiatric history as a risk factor, more recent large-scale epidemiologic studies did [4]. Other risk factors for neuropsychiatric symptoms are also damaged blood-brain barrier and hypoalbuminemia. All administration routes may be associated with neuropsychiatric adverse effects (including intra-nasal, topical, epidural or intra-articular administration). Ninety percent of patients will recover within 6 weeks (it may take longer for cognitive symptoms) [2].

In contrast, in some cases, positive effects such as improvement of depressive disorders without occurrence of mania as well as improvement of cognitive symptoms related to the underling disease were observed during corticosteroid treatment. Moreover, glucocorticoid treatment reduces fearpotentiated startle and post traumatic stress disorders risk after trauma, perhaps by enhancing extinction and extinction retention [5].

Treatment of neuropsychiatric side effects includes adjusting/discontinuing the corticosteroid treatment or prescribing psychotropic medications (mostly antipsychotics followed by mood stabilizers such as lithium, sodium valproate, lamotrigine, benzodiazepines or antidepressants). Psychoeducation of patients receiving corticosteroids plays also an important role.

The mechanism involved in these side effects remains poorly understood. In healthy subjects, cognitive dysfunctions as well as psychiatric symptoms were observed with corticosteroid or hydrocortisone treatment. Wolkowitz et al. [6] reported depression, mood elevation, irritability, insomnia, excessive talkativeness, increased appetite or, an inability to filter out distracting information in healthy controls receiving prednisone $(80 \mathrm{mg} /$ day $)$. Reduction in hippocampal volume occurs even during brief exposure to corticosteroids, and hippocampal changes can be blocked by phenytoin. Some drug-drug interactions (e.g., through inhibition of the CYP-3A4 cytochrome) may increase corticosteroid levels and favor adverse effects. Brain lesions associated with numerous systemic diseases may increase the risk of neuropsychiatric adverse effects. Corticosteroids, which regulate the activity of the raphe-hippocampal serotoninergic pathway, may affect the serotoninergic system and therefore increase the risk for anxiety or depressive symptoms. Interestingly in healthy subjects, haloperidol and, to a lesser extent, aripiprazole can directly regulate the HPA axis and immune system through a pharmacological action via D2 receptor antagonism [7]. In animal models, corticoids are associated with apoptosis and neuronal death. Furthermore, corticosteroids induce an increased release of glutamate that induces neuronal toxicity due to accumulation effect [6]. Preclinical and clinical research indicates that excess corticosteroid is associated with adverse effects on the hippocampus. Animal model data suggest that N-methylD-aspartate (NMDA) receptor antagonists (memantine) or glutamate release inhibitors (phenytoin or lamotrigine) may block corticosteroid effect on the hippocampus [8]. These compounds might be useful to prevent adverse effects of corticosteroid treatments.

Finally, the effects of glucocorticoids are mediated by both genomic (with a slow onset) and non-genomic (with a rapid onset and a short duration) mechanisms. Genomic mechanisms implicate the activation and/or repression of specific genes encoding anti- and pro-inflammatory proteins. The non-genomic mechanisms of glucocorticoid action remain poorly understood [9]. Polymorphisms of the GR gene (nuclear receptor subfamily three, group $\mathrm{C}$, member 1:NR3C1) are known to be associated with variations in the GR function [10]. The activated GR/GC complex exerts its anti-inflammatory and immunosuppressive effects at the nucleus level, directly (via DNA binding) or indirectly (via protein interaction), by decreasing expression of pro-inflammatory genes (transrepression), or increasing expression of anti-inflammatory genes (transactivation). The hypothesis that the anti-inflammatory properties of glucocorticoids are mostly due to transrepression of synthesis of inflammatory mediators, whereas most of their adverse effects are associated with transactivation of genes involved in metabolic processes, led to the development of GR ligands which cause a receptor conformation preferring a GR/protein interaction and not a GR/DNA binding-dependent mechanism, so-called selective glucocorticoid receptor agonists (SEGRAs) [9]. Moreover, GR (but not MR) genetic variations accounted for a significant amount of variance in mean cortisol levels and severity of psychosis or cognitive dysfunction in depression [10]. Therefore, genetic alterations in this gene may account for some degree of inter-individual variability in the glucocorticoid response and steroid-related toxicity. This area of interest, which has been developed in other medical fields where metabolic adverse effects of corticosteriods are observed, is under-researched in psychiatry.

\section{References}

1. Soria V, González-Rodríguez A, Huerta-Ramos E, Usall J, Cobo J, Bioque M, Barbero JD, García-Rizo C, Tost M, Monreal JA, PNECAT Group, Labad J (2018) Targeting hypothalamic-pituitaryadrenal axis hormones and sex steroids for improving cognition in major mood disorders and schizophrenia: a systematic review 
and narrative synthesis. Psychoneuroendocrinology 93:8-19. https ://doi.org/10.1016/j.psyneuen.2018.04.012

2. Dubovsky AN, Arvikar S, Stern TA, Axelrod L (2012) The neuropsychiatric complications of glucocorticoid use: steriod psychosis revisited. Psychosomatics 53:103-115

3. Lewis DA, Smith RE (1983) Steroid-induced psychiatric syndromes. A report of 14 cases and a review of the literature. J Affect Disord 5(4):319-332

4. Morrow SA, Barr J, Rosehart H, Ulch S (2015) Depression and hypomania symptoms are associated with high dose corticosteroids treatment for MS relapses. J Affect Disord 187:142-146. https://doi.org/10.1016/j.jad.2015.08.040

5. Rasmusson AM, Marx CE, Pinelesa SL, Loccie A, Scioli-Salter ER, Nillnia YI, Liang JJ, Pinnae G (2017) Neuroactive steroids and PTSD treatment. Neurosci Lett 649:156-163

6. Wolkowitz OM, Rubinow D, Doran AR, Breier A, Berrettini WH, Kling MA (1990) Prednisone effects on neurochemistry and behavior. Preliminary findings. Arch Gen Psychiatry 47:963-968

7. Handley R, Mondelli V, Zelaya F, Marques T, Taylor H, Reinders AATS, Chaddock C, McQueen G, Hubbard K, Papadopoulos A,
Williams S, McGuire P, Pariante C, Dazzan P (2016) Effects of antipsychotics on cortisol, interleukin-6 and hippocampal perfusion in healthy volunteers. Schizophr Res 174(1-3):99-105. https ://doi.org/10.1016/j.schres.2016.03.039

8. Brown ES, Sayeda N, Choic C, Tustisond N, Robertsd J, Yassad MA, Van Enkevorta E, Nakamuraa A, Ivlevaa EI, Sunderajana P, Khanb DA, Vazquez M, McEwen B, Kulikovaa A, Frola AB, Holmesa T (2019) A randomized, double-blind, placebo-controlled trial of lamotrigine for prescription corticosteroid effects on the human hippocampus. Eur Neuropsychopharmacol 29:376-383

9. Schijvens AM, Heine R, de Wildt SN, Schreuder MF (2019) Pharmacology and pharmacogenetics of prednisone and prednisolone in patients with nephrotic syndrome. Pediatr Nephrol 34:389-403

10. Keller J, Gomez R, Williams G, Lembke A, Lazzeroni L, Murphy GM, Schatzberg AF (2017) HPA axis in major depression: cortisol, clinical symptomatology, and genetic variation predict cognition. Mol Psychiatry 22(4):527-536. https://doi.org/10.1038/ mp.2016.120 